\title{
THE KOTTAMIA OBSERVATORY AND OTHER ASPECTS OF ASTRONOMY IN EGYPT
}

\author{
A.M.I.Osman \\ National Research Institute of \\ Astronomy and Geophysics \\ Helwan-Cairo, Egypt
}

The 74-inch $(1.8 \mathrm{~m})$ telescope of Kottamia Observatory is the largest telescope in North Africa and the Middle East. It stands on a plateau $485 \mathrm{~m}$ above sea-level and $70 \mathrm{~km}$ to the east of Cairo. The latitude is $25^{\circ} \quad 55^{\prime} .9 \mathrm{~N}$ and the longitude $31^{\circ} 49^{\prime} .5 \mathrm{E}$. The telescope is of conventional type, having Newtonian (f/4.9), Cassegrain (f/18) and coudé (f/28.9) foci; it was built by Grubb-Parsons (U.K.). The main mirror is of Duran 50 glass, and has a clear aperture of 74 inches $(1.8 \mathrm{~m})$ and a focal length, for its paraboloidal surface, of 360 inches $(9.2 \mathrm{~m})$. The secondary mirrors are of fused quartz. The telescope is provided with a Newtonian camera, a single-channel photoelectric photometer, a Cassegrain spectrograph comprising two cameras with dispersions of $100 \mathrm{Amm}^{-1}$ and $48 \mathrm{Amm}^{-1}$ at $4800 \mathrm{~A}$ and a coudé spectrograph with two cameras allowing dispersions of $20 \mathrm{Amm}^{-1}$ and $6 \mathrm{Amm}^{-1}$. A four-component correcting lens has been supplied to be mounted at the Newtonian focus, in order to reduce the effect of coma on photographic observations.

Since the telescope went into operation in 1964, many observations have been made and used in research work, for some of which M.Sc. and Ph.D. degrees were awarded. Extensive programmes of cooperation with other similar observatories and institutes all over the world were undertaken in several interesting lines of research.

In 1989 , a serious problem with the surface of the main mirror was encountered. When we tried to re-aluminize the surface in the usual way, it would not accept the film. The new film was weak and did not reflect enough light. Further attempts led to no improvement. Experts told us that the poor film could be attributed to some contamination of the surface. Analysis by Zeiss showed the existence of significant surface roughness, suggesting that repeated cleaning of the surface with acid had leached it. Surface scattering was thus increased, and the adhesion of the coating reduced. The aluminizing system might also have been a cause. We have evaluated the whole situation regarding the mirror and the auxiliary instruments, which have not been updated in the more than thirty years that the telescope has been in use. We shall 
undertake an extensive upgrading programme for the 74 -inch $(1.8 \mathrm{~m})$ telescope including:
A Zerodur primary mirror
A large-format (1024 x 1024 pix) liquid-nitrogen cooled CCD camera
Fast grating spectrograph
Updating the MDM6 microdensitometer with a new software package.

The main problem of such an upgrading programme is our serious shortage of technical staff. We need well-trained persons to run and to maintain the advanced technological equipment, such as CCD cameras, and who know how to use the appropriate software packages. We urgently need to be able to send people to observatories where they can be trained, and grants for training at any observatory or institution would be very welcome.

Nowadays, it is very difficult for any country in Africa or the Middle East to run a big telescope. We will be very happy to welcome any cooperation or agreement with any neighbouring country to use our telescope either for training or for observing. It would be good if international organizations could support our Observatory to serve as a centre for observations and training for all countries from Africa and the Middle East. 